\title{
Deep penetrating photoacoustic tomography in biological tissues
}

Geng Ku, Xueding Wang, Xueyi Xie, George Stoica, Lihong V. Wang

Geng Ku, Xueding Wang, Xueyi Xie, George Stoica, Lihong V. Wang, "Deep penetrating photoacoustic tomography in biological tissues," Proc. SPIE 5697, Photons Plus Ultrasound: Imaging and Sensing 2005: The Sixth Conference on Biomedical Thermoacoustics, Optoacoustics, and Acoustooptics, (25 April 2005); doi: 10.1117/12.588798

SPIE. Event: SPIE BiOS, 2005, San Jose, CA, United States 


\title{
Deep penetrating photoacoustic tomography in biological tissues
}

\author{
Geng $\mathrm{Ku}^{\mathrm{a}}$, Xueding Wang ${ }^{\mathrm{a}}$, Xueyi Xie ${ }^{\mathrm{a}}$, George Stoica ${ }^{\mathrm{b}}$ and Lihong V. Wang ${ }^{\mathrm{a}}$ \\ a. Optical Imaging Laboratory, Department of Biomedical Engineering, Texas A\&M University, \\ 3120 TAMU, College Station, TX 77843-3120 \\ b. Department of Pathobiology, Texas A\&M University, College Station, TX 77843-5547
}

\begin{abstract}
Photoacoustic tomography (PAT) in a circular scanning configuration was developed to image the deeply embedded optical heterogeneity in biological tissues. Based on the intrinsic contrast between blood and chicken breast muscle, an embedded blood object that was $5 \mathrm{~cm}$ deep in the tissue was detected using pulsed laser light at a wavelength of 1064 $\mathrm{nm}$. Compared with detectors for flat active surfaces, cylindrically focused ultrasonic transducers can reduce the interference generated from the off-plane photoacoustic sources and make the image in the scanning plane clearer. While the optical penetration was optimized with near-infrared laser pulses of $800 \mathrm{~nm}$ in wavelength, the optical contrast was enhanced by indocyanine green (ICG) whose absorption peak matched the laser wavelength. This optimized PAT was able to image fine objects embedded at a depth of up to $5.2-\mathrm{cm}$, which is 6.2 times the $1 / \mathrm{e}$ optical penetration depth, in chicken breast muscle, at a resolution of $<\sim 750$ microns with a sensitivity of $<7$ pmol of ICG in blood. The resolution was found to deteriorate slowly with increasing imaging depth.
\end{abstract}

Keywords: photoacoustic tomography, medical imaging, biological tissue, breast imaging, spatial resolution, image reconstruction, optical contrast agent.

\section{INTRODUCTION}

Photoacoustic tomography (PAT), based on the measurement of laser-induced ultrasonic waves, is a non-ionizing imaging modality for visualizing biological tissues with high optical contrast and high ultrasonic resolution. For some specified wavelength of light, the absorption coefficient of blood can be 10 times higher than that of its surrounding tissues, ${ }^{1}$ which results in excellent intrinsic tissue contrast. The photoacoustic imaging modality has been successfully applied to imaging vasculature structures and tumor angiogenesis a few $\mathrm{mm}$ under the skin. ${ }^{2-4}$ Oraevsky et al. studied the imaging sensitivity of PAT on deep embedded objects using tissue phantoms, ${ }^{5}$ and they reported a PAT image of a ductal carcinoma at a depth of $1.1 \mathrm{~cm}$ from the breast surface.$^{6,7}$ Due to the overwhelming scattering effect of light in biological tissues, light intensity, and hence the photoacoustic signal-to-noise ratio (SNR), decreases with depth exponentially with a decay constant of a few $\mathrm{mm}$. The light intensity attenuation can be minimized by carefully choosing the excitation laser wavelength such that the optical penetration in the tissues is maximized. Near-infrared (NIR) has a low absorption coefficient and a relatively low scattering coefficient in biological tissues and can, consequently, provide deep penetration at the expense of reduced photoacoustic signal strength ${ }^{8}$. However, photoacoustic signals can be enhanced by using a matched optical contrast agent. Indocyanine green (ICG) has a strong absorption peak in the NIR region and can serve such a purpose. ICG is widely applied in clinical applications such as cardiac output monitoring, ${ }^{10}$ hepatic function studies, ${ }^{11}$ angiography in ophthalmology, ${ }^{12}$ and tumor detection. ${ }^{13}$

Photoacoustic imaging is based on ultrasonic propagation and detection, which means that it has the same good spatial resolution as pure ultrasound imaging. The geometry of the region over which the ultrasonic transducer is scanned determines the imaging mode and the reconstruction algorithms. For the detection of layered structures of tissues or tumors under human epidermis, the ultrasonic transducer must be placed on the skin along the laser beam to accomplish detection in vivo. Karabutov and Oraevsky et al. ${ }^{14,15}$ presented photoacoustic imaging with axial resolution up to $15 \mu \mathrm{m}$. The imaging of small deeply embedded tumors, such as breast tumors, was also studied by Esenaliev et al. ${ }^{16}$ and Oraevsky et al. ${ }^{17}$ Hoelen $e t$ al. employed a transducer array on a plane to detect induced signals from vascular systems. ${ }^{18}$ The image was reconstructed using a delay-and-sum algorithm, where the depth resolution was better than 20

\footnotetext{
* To whom all correspondence should be addressed: Telephone: 979-847-9040; Fax: 979-845-4450; Electronic mail: LWang@tamu.edu; URL: http://oilab.tamu.edu.
} 
$\mu \mathrm{m}$, and the lateral resolution was better than $200 \mu \mathrm{m}$. The detector can also scan over a circular track, and reconstruction algorithms have been developed to use the signals from the scan to form an image. Examples of current reconstruction algorithms include the weighted delay-and-sum method, the optimal statistical approach, ${ }^{19}$ and the Radon transform in the far-field approximation. ${ }^{20}$ Only recently have exact reconstructions been studied theoretically and reconstruction algorithms for various regular geometries derived. ${ }^{22-25}$

The generation, propagation, and detection of induced acoustic signals can be described by thermal expansion, wave equations and bandwidth filtering, respectively. ${ }^{26}$ By directing the laser pulse on a test sample, the absorbed optical energy in the tissue is transformed into thermal energy, which is then, due to thermo-elastic expansion, converted into mechanical stress. If the energy deposition occurs rapidly in a time period that is much less than the thermal relaxation time and the stress relaxation time, the local pressure rise after heating can be derived as $\Delta p=\frac{\beta c^{2}}{C_{p}} E_{a}$, where $\Delta p$ is the pressure rise; $\beta$ is the isobaric volume expansion coefficient; $c$ is the speed of sound; $C_{p}$ is the specific heat; and $E_{a}$ is the absorbed optical energy density. The behavior of photoacoustic waves has been studied by Diebold and Gusev et al. based on the following non-homogenous wave equation: ${ }^{27,28}$

$$
\nabla^{2} p(\mathbf{r}, t)-\frac{1}{c^{2}} \frac{\partial^{2} p(\mathbf{r}, t)}{\partial t^{2}}=-\frac{\beta}{C_{p}} \frac{\partial H(\mathbf{r}, t)}{\partial t}
$$

where $p(\mathbf{r}, t)$ is the acoustic pressure at time $t$ and position $\mathbf{r}$ and $H(\mathbf{r}, t)$ is the heat function of the optical energy deposited in the tissues per unit volume per unit time, which can be expressed as

$$
H(\mathbf{r}, t)=A(\mathbf{r}) I(t)
$$

where $A(\mathbf{r})$ describes the optical energy deposition within the tissues at position $\mathbf{r}$ (structure of tissues); and $I(t)$ describes the shape of the irradiation pulse, which can be further expressed as $I(t)=\delta(t)$ for impulse heating.

The purpose of photoacoustic tomography is to reconstruct the distribution of the optical absorption $A(\mathbf{r})$ in the tissues from a set of measured acoustic signals $p(\mathbf{r}, t)$. For a cylindrical scanning configuration, the exact inverse solution can be derived. In most practical image reconstructions, the algorithm can be simplified to shorten the reconstruction time. If the detection radius $r_{0}$ is much longer than the wavelength that corresponds to the central frequency of the detection device (far-field detection), we can assume $|k| r_{0}>>1$ and simplify the exact inverse solution to the form of ${ }^{29}$

$$
A(\mathbf{r})=-\left.\frac{\beta}{2 \pi c^{4} C_{p}} \iint_{S_{0}} d S_{0}(\cos \Theta) \frac{1}{t} \frac{\partial p\left(\mathbf{r}_{0}, t\right)}{\partial t}\right|_{t=\left|\mathbf{r}_{0}-\mathbf{r}\right| / c}
$$

where $S_{0}$ is the surface over which the detectors are scanned; $\Theta$ is the angle between the normal line of the detector and the vector from the detector to the reconstruction point.

\section{EXPERIMENTAL SETUP}

In Fig. 1, our experimental setup of deep penetrating PAT is shown. A pulse Ti:Sapphire laser (LOTIS TII, Symphotic), which is pumped by a Q-switched Nd:YAG laser at a repetition rate of $10 \mathrm{~Hz}$, provides $\sim 10$-ns NIR laser pulses at a wavelength of $800 \mathrm{~nm}$. The Ti:Sapphire and Q-switched Nd:YAG laser can provide $120 \mathrm{mj}$ and $1 \mathrm{~J}$ output energy at wavelengths of $800 \mathrm{~nm}$ and $1064 \mathrm{~nm}$, respectively. The laser beam is expanded by a concave lens, homogenized by a ground glass and then directed onto a sample made from chicken breast muscle tissue. The incident laser energy densities on the tissue surface are $\sim 7 \mathrm{~mJ} / \mathrm{cm}^{2}$ and $\sim 60 \mathrm{~mJ} / \mathrm{cm}^{2}$ for the above two near infrared lights, respectively. The PAT system has four independent detection channels that can receive photoacoustic signals through multiple ultrasonic transducers simultaneously. Four ultrasonic transducers (V323/2.25-MHz, V383/3.5-MHz, V157/5$\mathrm{MHz}$, and V312/10-MHz, Panametrics), with nominal bandwidths ranging from 50\% to $80 \%$ of their specified central frequencies, are used for detection in four different frequency ranges. The diameters of their active elements are $0.6 \mathrm{~cm}$, $1 \mathrm{~cm}, 0.3 \mathrm{~cm}$ and $0.6 \mathrm{~cm}$ for the $2.25-\mathrm{MHz}, 3.5-\mathrm{MHz}, 5-\mathrm{MHz}$ and $10-\mathrm{MHz}$ transducers, respectively. The 3.5-MHz 
transducer is cylindrically focused in the vertical dimension with a focal length of $3.2 \mathrm{~cm}$ while the others have flat active surfaces. The transducers convert the photoacoustic pressure into piezoelectric signals. The signals are subsequently amplified by amplifiers (ZFL-500LN, Mini-Circuits; 5072PR, Panametrics), bandpass-filtered by our recently homemade filters, and finally recorded by an oscilloscope (TDS-540A, Tektronix). The sample and the transducers are immersed in a tank of water for coupling the photoacoustic waves to the transducers. The transducers are driven by a step motor to scan horizontally along a $10-\mathrm{cm}$ diameter circle around the sample with a step size of 1.5 degrees. A personal computer is used to control the scanning and data acquisition.

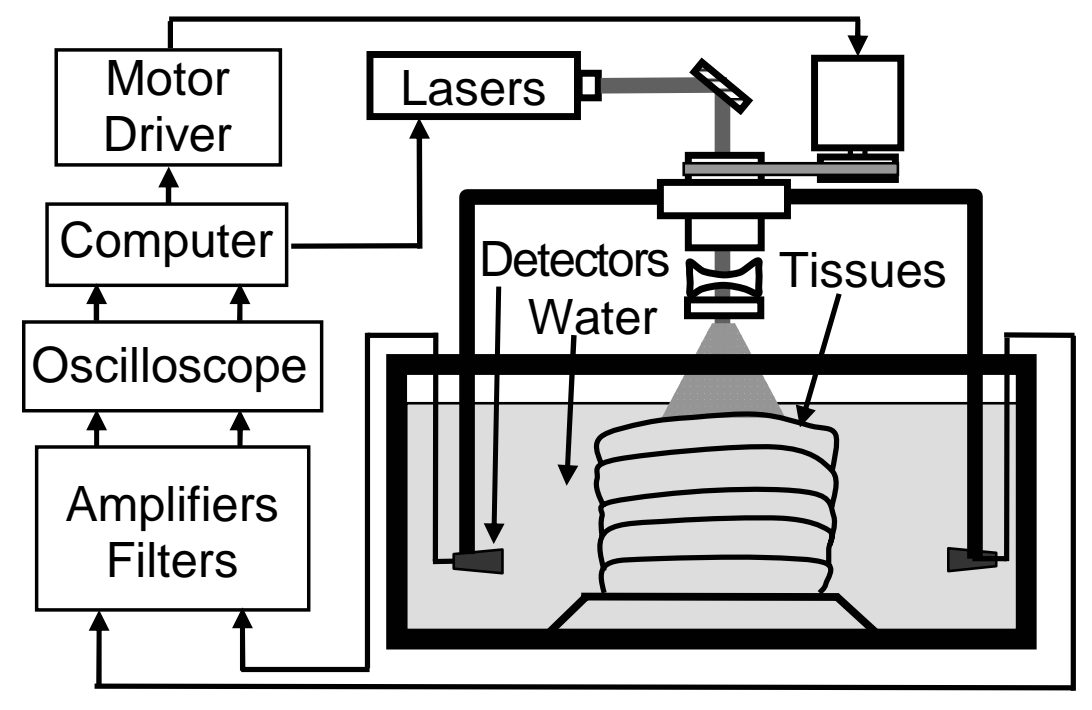

Figure 1. Experimental setup of deep penetrating photoacoustic tomography.

\section{RESULTS AND DISCUSSION}

Generally, blood in the vasculature has stronger optical absorption than in the adjacent tissues. Imaging based on this intrinsic contrast is attractive and highly useful in medical imaging applications. Whole blood's absorption coefficient for infrared light at a wavelength of $1.06 \mu \mathrm{m}$ is 4 to $5 \mathrm{~cm}^{-1}$. In order to image deep vasculature using intrinsic contrast, we chose $1.06 \mu \mathrm{m}$ laser pulses as the exciting light in our experiment. This NIR light is deeply penetrating in most biological tissues. Furthermore, the output of fundamental oscillation from the Nd:YAG laser in our lab can provide full power illumination.

Using NIR laser pulses at the $1.06 \mu \mathrm{m}$ wavelength, we imaged the blood object embedded in porcine fat tissue as shown in Fig. 2. Seven pieces of porcine fat tissue were sequentially stacked up to increase the imaging depth as shown in the left picture in Fig. 2. Laser light illuminated the test sample from its top. The object was made by pouring raw rat blood into a transparent plastic tube with an inner diameter of $1.5 \mathrm{~mm}$ and embedding it in the porcine fat tissue. The photograph of the cross section of the embedded blood object is on the top right of Fig. 2. The PAT image of the blood object at a depth of $5 \mathrm{~cm}$ is shown on the bottom right. 


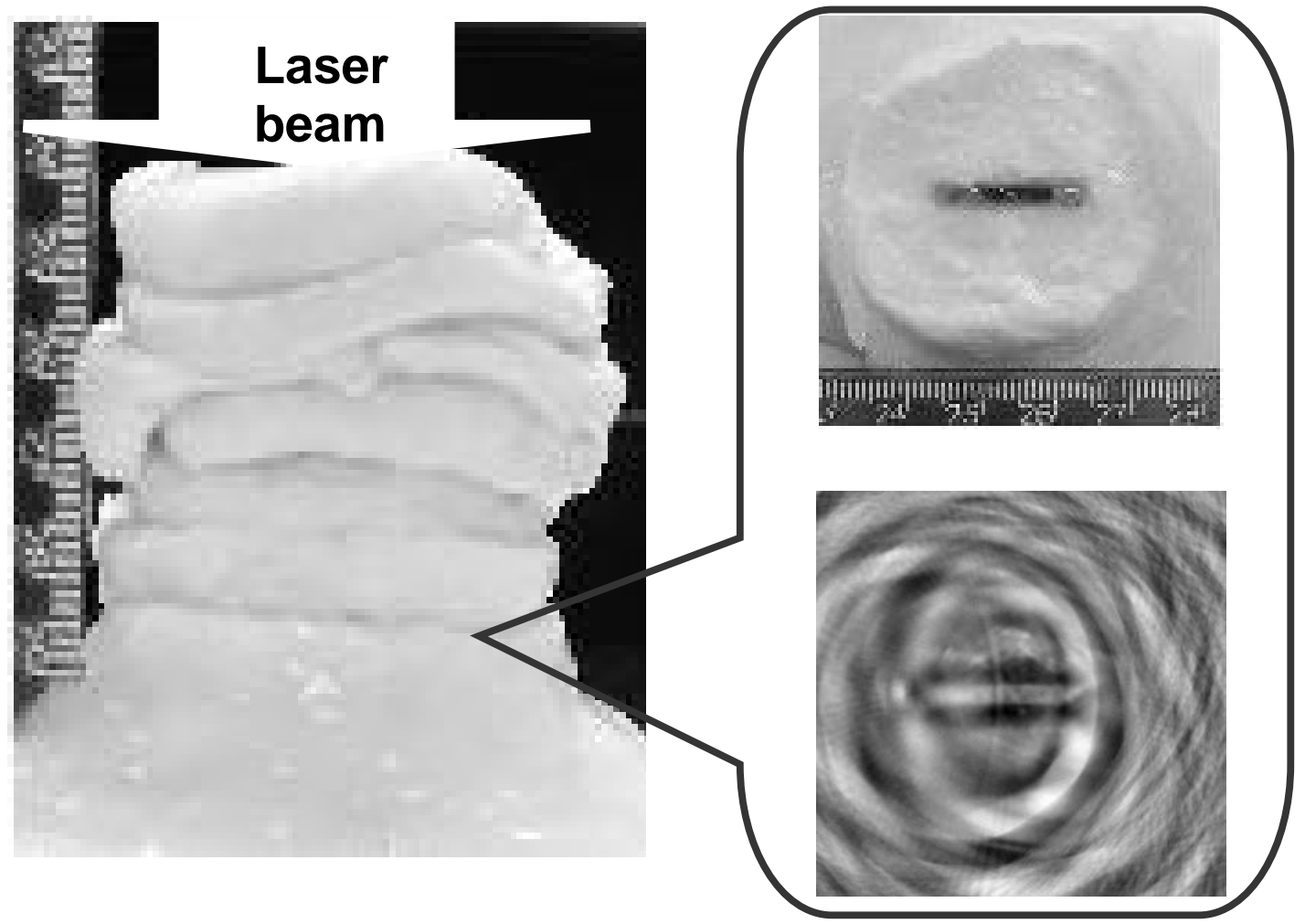

Figure 2. Blood sample in porcine fat and its 2D PAT images using a laser light of $1.06-\mu \mathrm{m}$ wavelength. Left: photograph of the entire sample. Top right: photograph of the imaged cross section of porcine fat in which raw blood is embedded, Bottom right: two-dimensional photoacoustic images acquired with a $2.25-\mathrm{MHz}$ ultrasonic transducer at depths of $5 \mathrm{~cm}$.

As each porcine fat sample was added, the blood embedded cross section of the porcine fat tissue was simultaneously scanned by four transducers. The PAT images at depths of $0.9 \mathrm{~cm}, 3.6 \mathrm{~cm}, 4.3 \mathrm{~cm}$ and $5 \mathrm{~cm}$ achieved by 2.25-MHz transducer and 3.5-MHz transducer are shown in Fig. 3(a) to (d) and Fig. 4(a) to (d), respectively. The blood object was imaged clearly at a depth of $0.9 \mathrm{~cm}$. In porcine fat, there are some vessels of an aboriginal nature or other optical absorbing tissues, which absorb laser energy and generate photoacoustic signals. When the absorbers are located above the transducers' scanning plane, (in which the blood object is embedded), and near the laser illuminated surface, more laser energy will be absorbed and stronger photoacoustic signals will be generated. Since the photoacoustic sources are not seated in the horizontal imaging plane, they are blurred in the reconstructed images where the blood column is well focused. The farther the aboriginal nature absorbers are away from the scanning plane, the more they are blurred, even appearing as circles in some images. According to the radius of the circles on the PAT images, this interference was estimated to be coming from a location near the laser illuminated tissue surface. When the imaging depth increased, the interference became more obvious and meddlesome in the image, and the embedded blood object could only be recognized from its geometrical shape. This phenomenon is obvious in the images obtained by the flat transducer (2.25-MHz, $6 \mathrm{~mm}$ in diameter) shown in Fig. 3. 

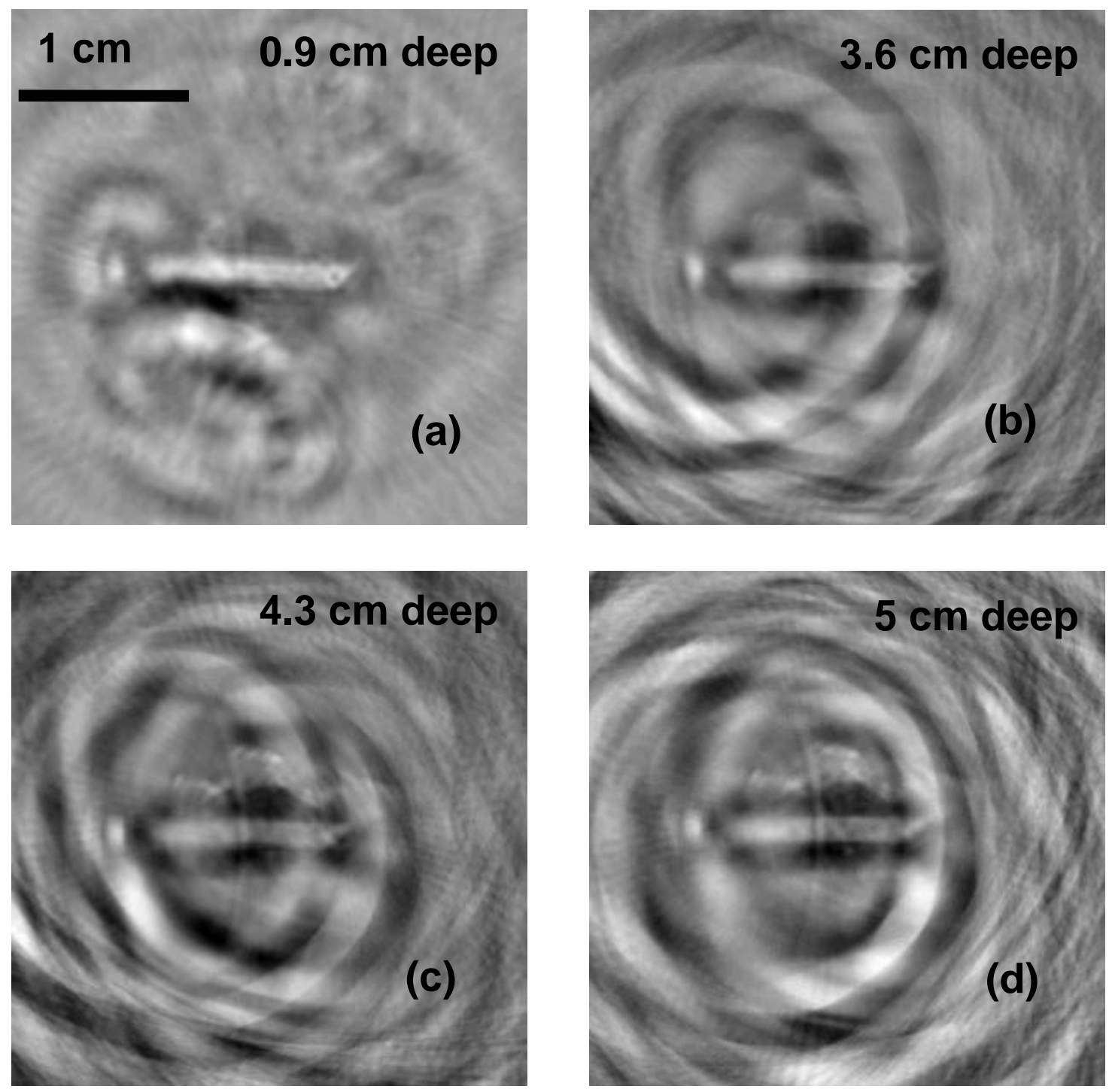

Figure 3. Two-dimensional photoacoustic images of blood object in porcine fat acquired with a 2.25MHz ultrasonic transducer at depths of (c) $0.9 \mathrm{~cm}$, (d) $3.6 \mathrm{~cm}$, (e) $4.3 \mathrm{~cm}$, and (f) $5 \mathrm{~cm}$, respectively.

In the images detected by the horizontally cylindrically focused transducer $(3.5-\mathrm{MHz}, 10 \mathrm{~mm}$ in diameter) are shown in Fig. 4, the interference is not serious because the signals generated from the induced photoacoustic sources beyond the focal zone of the detector are cancelled out. The images shown in Fig. 4 are cleaner than those in Fig. 3. Even when the imaging depth increased to $5 \mathrm{~cm}$, the blood column is obscure but still discernible.

The laser power density at the tissue surface was measured to be $63 \mathrm{~mW} / \mathrm{cm}^{2}$ at a wavelength of $1.06 \mu \mathrm{m}$. The laser light transmitted through the tissues was also measured using a power meter (SmartSensor 30V, Coherent). The photoacoustic signal was proportional to the laser light intensity; therefore, the photoacoustic signal amplitude could be used to scale the light attenuation. The effective attenuation coefficient of the porcine fat tissue was fitted to be around $2.5 \mathrm{~cm}^{-1}$ and $2.1 \mathrm{~cm}^{-1}$ according to both the transmitted light power measurement and the photoacoustic signal attenuation. Therefore, the $5 \mathrm{~cm}$ imaging depth is approximately 10 times the 1/e optical penetration depth, corresponding to $43-\mathrm{dB}$ attenuation from the incident to the transmitted optical energy density. 

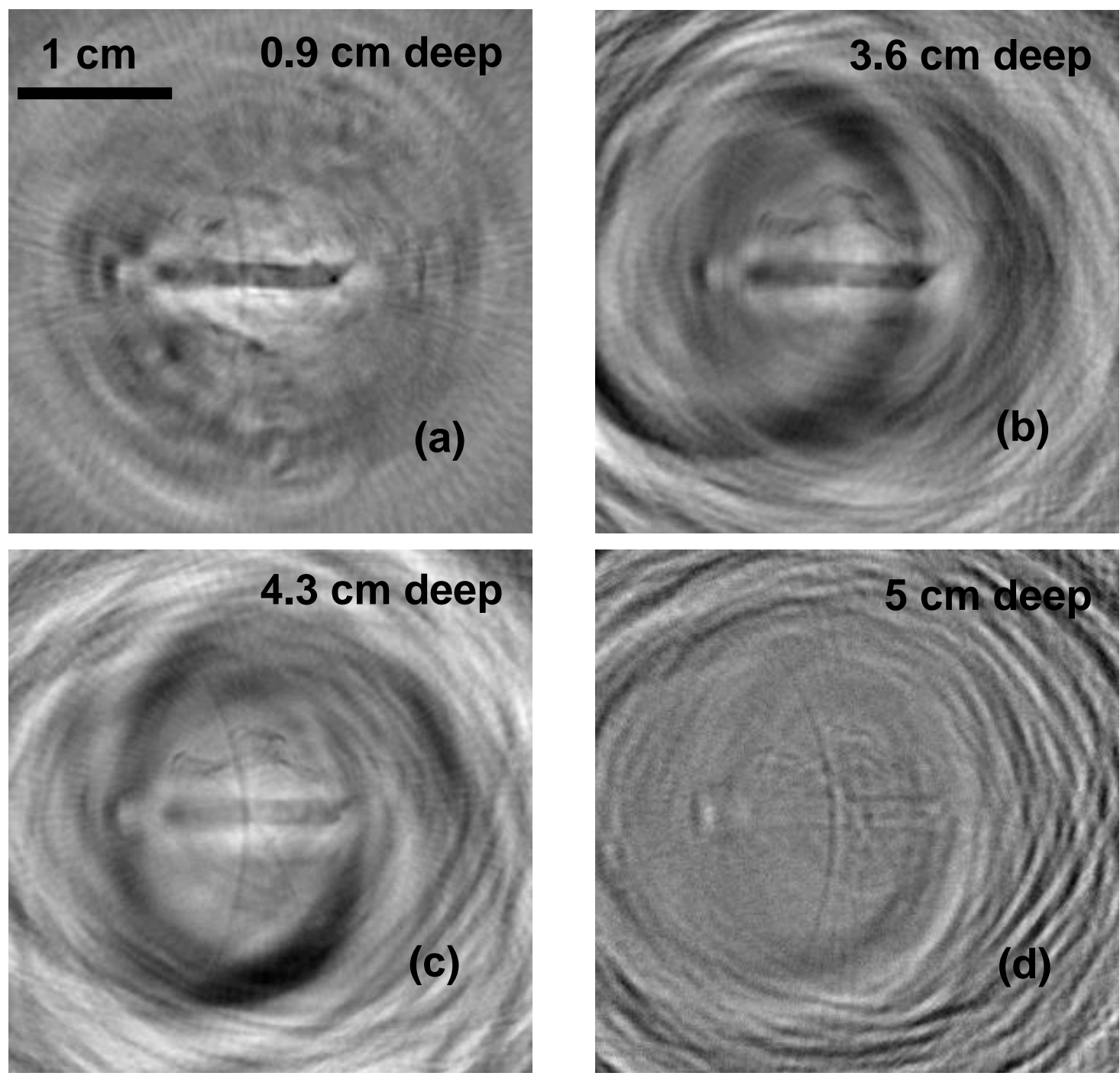

Figure 4. Two-dimensional photoacoustic images of a blood object in porcine fat acquired with a 3.5$\mathrm{MHz}$ ultrasonic transducer at depths of (c) $0.9 \mathrm{~cm}$, (d) $3.6 \mathrm{~cm}$, (e) $4.3 \mathrm{~cm}$, and (f) $5 \mathrm{~cm}$, respectively.

Using ICG to enhance photoacoustic signals and hence to image deep fine vascular structures was investigated. Three pairs of transparent plastic tubes of a $300 \mu \mathrm{m}$ inner diameter each were embedded in chicken breast tissues of a $\sim 7.5 \mathrm{~cm}$ diameter in their transverse cross sections [Fig. 5(b)]. The first pair of tubes was filled with anticoagulated whole rat blood; the second with ICG solution in distilled water (323 $\mu \mathrm{M})$; and the last with ICG in rat blood (129 $\mu \mathrm{M})$. Those centripetally arranged objects were placed in the transducers' scanning plane. At an 800-nm wavelength, the optical absorption coefficient is $\sim 4-5 \mathrm{~cm}^{-1}$ for whole blood, $16 \mathrm{~cm}^{-1}$ for ICG in water and $43 \mathrm{~cm}^{-1}$ for ICG in blood. Four pieces of chicken breast muscle were stacked up on the objects [Fig. 5(a)]. When excited by laser light illuminated from the top surface, the embedded objects emitted photoacoustic waves that propagated through the medium. The photoacoustic signals were picked up by the circularly stationed transducers and fed into the image reconstruction based on a back projection algorithm. ${ }^{31}$ 


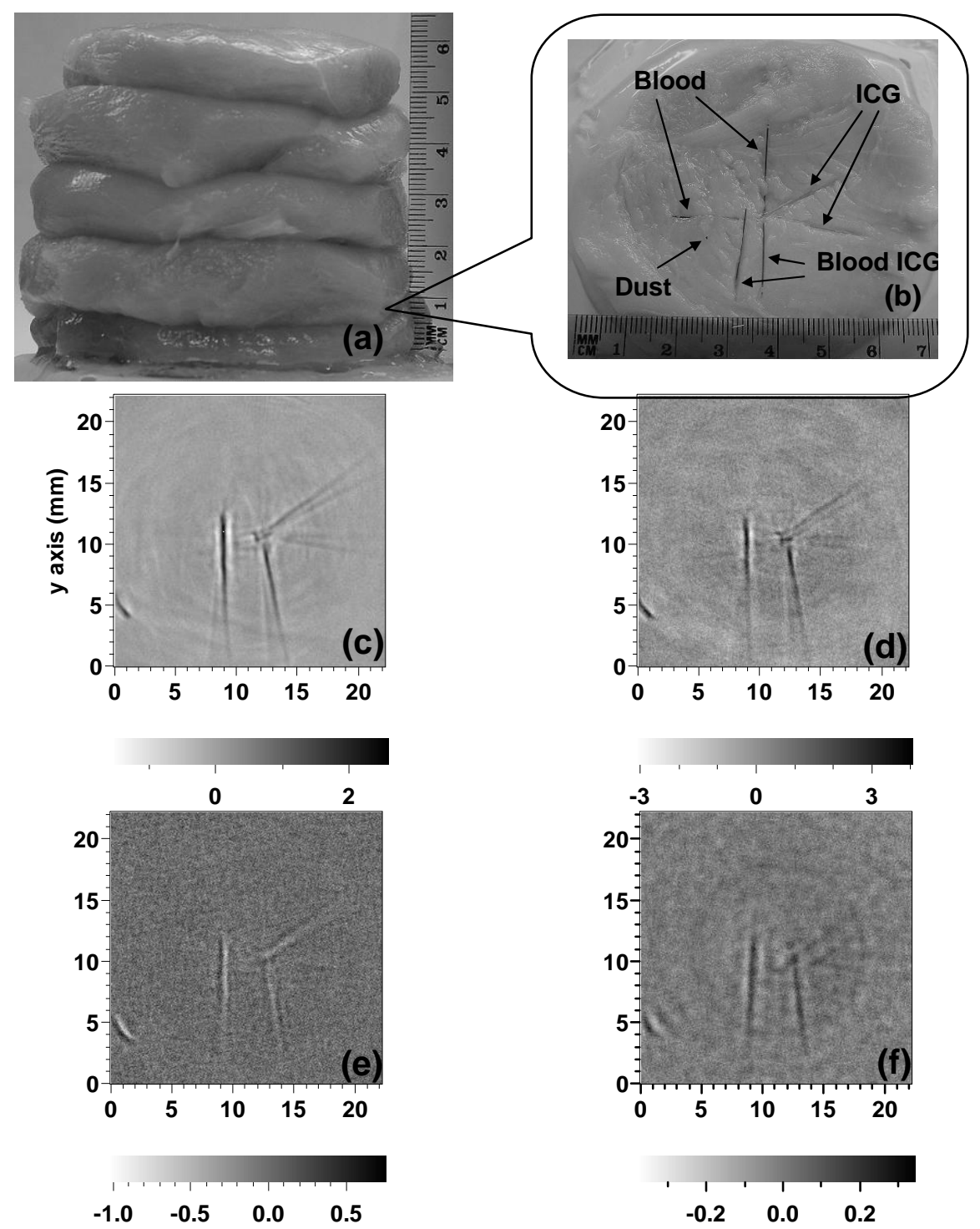

Figure 5. Two-dimensional photoacoustic imaging at various depths. (a) Photograph of the cross sections of chicken breast tissue in which objects containing blood or ICG, or both, are embedded. (b) Photograph of the entire sample. Two-dimensional photoacoustic images acquired with a $3.5-\mathrm{MHz}$ ultrasonic transducer at depths of (c) $1.3 \mathrm{~cm}$, (d) $2.6 \mathrm{~cm}$, (e) $4.2 \mathrm{~cm}$, and (f) $5.2 \mathrm{~cm}$, respectively.

The tissue's cross section containing the embedded objects was imaged with four pieces of chicken breast tissue sequentially stacked up, which made the embedded objects $1.3 \mathrm{~cm}, 2.6 \mathrm{~cm}, 4.2 \mathrm{~cm}$, and $5.2 \mathrm{~cm}$ from the laser illuminated tissue surface, respectively. Figures 5(c) to (f) show the photoacoustic images obtained at each depth by the 3.5-MHz cylindrically focused transducer. A black dust grain in the objects' plane is also clearly imaged. The two whole blood tubes are liminal at the imaging depth of $1.3 \mathrm{~cm}$ and become unrecognizable when the depth further increases. The ICG solution in water is discernible at depths up to $5.2 \mathrm{~cm}$. The two ICG-enhanced blood tubes have the strongest photoacoustic signals and are clearly imaged throughout. Since the photoacoustic strength is proportional to the absorbed optical energy density in an optical absorber, the photoacoustic signal strengths of the above three kinds of objects at the same depth reflect the differences in their optical absorption properties. To improve the image quality, we averaged the photoacoustic signals 50 times at a depth of $1.3 \mathrm{~cm}, 100$ times at $2.6 \mathrm{~cm}$, and 400 times each at $4.2 \mathrm{~cm}$ and $5.2 \mathrm{~cm}$.

The light intensities that transmit through the four tissue thicknesses were detected using a photodiode detector 
(DET110, Thorlabs). The effective attenuation coefficient of the chicken breast tissue was fitted to be $1.2 \mathrm{~cm}^{-1}$ based on Beer's law. Therefore, the $5.2-\mathrm{cm}$ maximum imaging depth amounts to approximately 6.2 times the $1 / \mathrm{e}$ optical penetration depth, corresponding to a $27-\mathrm{dB}$ attenuation from the incident to the transmitted optical energy density.

We can estimate the sensitivity of our PAT on the detection of ICG based on the ICG concentration and the resolvable sample volume. At the 5.2-cm imaging depth, the resolvable sample volume is $\sim\left(\pi \times 0.3^{2} / 4\right) \times 0.75 \mathrm{~mm}^{3}$, where $0.3 \mathrm{~mm}$ is the diameter of the tubes and $0.75 \mathrm{~mm}$ is the overestimated resolution. Based on the concentration of ICG in the blood ICG mixture, the resolvable sample volume contains $\sim 7$ pmol of ICG. Because the spatial resolution is better than $0.75 \mathrm{~mm}$, the sensitivity is actually better than $7 \mathrm{pmol}$. Of course, the sensitivity is even better at shallower imaging depths. If the incident laser energy density is increased toward the MPE, the sensitivity can be further improved.

\section{CONCLUSION}

Three aspects of PAT were optimized to image deep tissue structures. Firstly, NIR laser light, which has deep penetration in biological tissues, was employed as the excitation source. A blood object embedded in $5 \mathrm{~cm}$ deep tissue was detected based on the tissue's intrinsic contrast. Secondly, an optical contrast agent, ICG, was applied to enhance the optical absorption of blood. In this way, fine vasculature deep in the tissues could be imaged with a good signal-tonoise ratio. Thirdly, multiple ultrasonic transducers were simultaneously used to balance the detection sensitivity and image resolution. It was demonstrated that fine tubes containing blood and ICG embedded at a depth greater than 5-cm in chicken breast tissue could be clearly imaged using PAT with a better than $750-\mu \mathrm{m}$ resolution. These results suggest that a sample of up to $10 \mathrm{~cm}$ in thickness could be imaged if both sides of the sample were illuminated. Therefore, nonionizing PAT can potentially image deep vasculature in the breast and other organs.

We thank M. Sivaramakrishnan and K. Song for lab assistance. This study is sponsored in part by the National Institute of Health grants R01 EB000712 and R01 NS46214 and the Texas ARP grant 000512-0063-2001. Wang's email is LWang@tamu.edu.

\section{REFERENCES}

1. $\quad$ S. L. Jacques and S. A. Prahl, "Absorption spectra for biological tissues," http://omlc.ogi.edu/classroom/ece532/class3/muaspectra.html, 2004.

2. R. G. M. Kolkman, E. Hondebrink, W. Steenbergen, F. F. M. de Mul, "In vivo photoacoustic imaging of blood vessels using an extreme-narrow aperture sensor," IEEE J. Sel. Top. Quantum Electron. Vol. 9, no 2, pp 343346, Mar.-Apr. 2003.

3. X. Wang, Y. Pang, G. Ku, X. Xie, G. Stoica, and L.-H. V. Wang, "Non-invasive laser-induced photoacoustic tomography for structural and functional imaging of the brain in vivo," Nat. Biotechnol. Vol.21, no 7, pp 803806, Jul 2003.

4. $\quad$ G. Ku, X. Wang, X. Xie, G. Stoica, L.-H. V. Wang, "Multiple-bandwidth photoacoustic tomography," Phys. Med. Biol. Vol. 49, no 7, pp. 1329-1338, Apr. 2004.

5. R. O. Esenaliev, A. A. Karabutov and A. A. Oraevsky, "Sensitivity of laser opto-acoustic imaging in detection of small deeply embedded tumors" IEEE J. Sel. Top. Quant. Vol.5, no. 4, pp. 981-988, Jul. 1999.

6. A. A. Karabutov, E. V. Savateeva, A. A. Oraevsky, "Optoacoustic tomography: New modality of laser diagnostic systems," LASER PHYSICS Vol. 13, no. 5, pp. 711-723, May 2003.

7. A. A. Oraevsky, E. V. Savateeva, S. V. Solomatin, A. A. Karabutov, V. G. Andreev, Z. Gatalica, T. Khamapirad, and P. M. Henrichs, in Biomedical Optoacoustics III, A. A. Oraevsky, ed., Proc. Vol.4618, pp. 81, 2002.

8. G. Ku, and L.-H. Wang, "Deep penetrating photoacoustic tomography in biological tissues enhanced with an optical contrast agent," Opt. Lett. 2005. (accepted)

9. X. Wang, G. Ku, M. A. Wegiel, D. J. Bornhop, G. Stoica, L.-H. V. Wang, "Noninvasive photoacoustic angiography of animal brains in vivo with near-infrared light and an optical contrast agent"Opt. Lett. Vol. 29, no. 7, pp730-732, Apr. 2004. 
10. Y.-L. He, H. Tanigami, H. Ueyama, T. Mashimo, and I. Yoshiya, "Measurement of blood volume using indocyanine green measured with pulse-spectrophotometry: Its reproducibility and reliability," Crit. Care Med. Vol. 26, no. 8, pp. 1446-1451,1998.

11. J. Caesar, S. Shaldon, L. Chiandussi, L. Guevara, and S. Sherlock, "The use of indocyanine green in the measurement of hepatic blood flow and as a test of hepatic function," Clin. Sci. Vol. 21, pp.43-57, 1961.

12. B. F. Hochheim, “Angiography of retina with indocyanine green,” Arch. Ophthalmol. Vol. 86, no. 5, pp. 564\&,1971.

13. M. M. Haglund, M. S. Berger, and D. W. "Enhanced optical imaging of human gliomas and tumor margins," Hochman, Neurosurgery Vol. 38, no. 2, pp. 308-317, Feb. 1996.

14. A. A. Karabutov, E. V. Savateeva, N. B. Podymova, and A. A. Oraevsky, "Backward mode detection of laserinduced wide-band ultrasonic ultrasonic transients with optoacoustic transducer," J. Appl. Phys., vol. 87, no. 4, pp. 2003-2014, Feb. 2000.

15. A. A. Karabutov, E. V. Savateeva, and A. A. Oraevsky, "Imaging of layered structures in biological tissues with opto-acoustic front surface transducer,” Proc. SPIE, vol. 3601, pp. 284-295, June 1999.

16. R. O. Esenaliev, A. A. Karabutov, and A. A. Oraevsky, "Sensitivity of laser opto-acoustic imaging in detection of small deeply embedded tumors," IEEE J. Sel. Top. Quant., vol. 5, no. 4, pp. 981-988, Aug. 1999.

17. R. O. Esenaliev, F. K. Tittel, S. L. Thomsen, B. Fornage, C. Stelling, A. A. Karabutov, and A. A. Oraevsky, "Laser optoacoustic imaging for breast cancer diagnostics: Limit of detection and comparison with X-ray and ultrasound imaging," Proc. SPIE, vol. 2979, pp. 71-82, Aug. 1997.

18. C.G.A. Hoelen, and F. F. M. de Mul, "Image reconstruction for photoacoustic scanning of tissue structures," Appl. Opt. vol. 39, no. 31, pp. 5872-5883, Nov. 2000.

19. Y. V. Zhulina, "Optimal statistical approach to optoacoustic image reconstruction,” Appl. Opt. vol. 39, no. 32, pp. 5971-5977, Nov. 2000.

20. R. A. Kruger, D. R. Reinecke, and G. A. Kruger, “Thermoacoustic computed tomography-technical considerations,” Med. Phys. vol. 26, no. 9, pp. 1832-1837, Sep. 1999.

21. R. A. Kruger, W. L. Kiser, K. D. Miller; H. E. Reynolds, D. R. Reinecke, G. A. Kruger, and P. J. Hofacker, “Thermoacoustic CT: imaging principles," Proc. SPIE, vol. 3916, pp. 150-159, May 2000.

22. K. P. Kostli, M. Frenz, H. Bebie, and H. P. Weber, "Temporal backward projection of optoacoustic pressure transients using Fourier transform methods," Phys. Med. Biol., vol. 46, no. 7, pp. 1863-1872, Jul. 2001.

23. M. Xu, and L.-H. Wang, "Time-domain reconstruction for thermoacoustic tomography in a spherical geometry," IEEE Trans. Med. Imag. vol. 21, no. 7, pp. 814-822, Jul. 2002.

24. Y. Xu, D. Feng, and L.-H. Wang, "Exact frequency-domain reconstruction for thermoacoustic tomography: I. Planar geometry,” IEEE Trans. Med. Imag. vol. 21, no. 7, pp. 823-828, Jul. 2002.

25. Y. Xu, M. Xu, L.-H. Wang, "Exact frequency-domain reconstruction for thermoacoustic tomography:II. Cylindrical geometry,” IEEE Trans. Med. Imag. vol. 21, no. 7, pp. 829-833, Jul. 2002.

26. G. Ku, L.-H. Wang, "Scanning thermoacoustic tomography in biological tissue," Med. Phys., vol. 27, no. 5 pp. 1195-1202, May 2000.

27. G. J. Diebold, T. Sun, and M. I. Khan, "Photoacoustic waveforms generated by fluid bodies," in Photoacoustic and Photothermal Phenomena III, edited by D. Bicanic, Springer-Verlag, pp. 263-269, Heidelberg, Berlin, 1992.

28. V. E. Gusev and A. A. Karabutov, Laser Optoacoustics. American Institute of Physics, New York: 1993. 
29. M. H. Xu, Yuan Xu and L.-H. V. Wang, "Time-domain reconstruction algorithms and numerical simulations for thermoacoustic tomography in various geometries," IEEE Trans. on Biomed. Eng., Vol. 50, pp.1086-1099, Sep. 2003.

30. M. L. J. Landsman, G. Kwant, G. A. Mook, W. G. Zijlstra, "Light -absorbing properties, stability, and spectral stabilization of indocyanine green,” J. Appl. Physiol. Vol. 40, no. 4, pp. 575-583, 1976.

31. M. Xu, L.-H. V. Wang, "Time-domain reconstruction for thermoacoustic tomography in a spherical geometry," IEEE Trans. Med. Imag. Vol. 21, no. 7, pp. 814-822, Jul. 2002. 